\title{
Acknowledgment to Reviewers of Multimodal Technologies and Interaction in 2020
}

\author{
Multimodal Technologies and Interaction Editorial Office
}

Citation: Multimodal Technologies and Interaction Editorial Office. Acknowledgment to Reviewers of Multimodal Technologies and Interaction in 2020. Multimodal Technol. Interact. 2021, 5, 4 . https://doi.org/10.3390/mti5010004

Published: 18 January 2021

Publisher's Note: MDPI stays neutral with regard to jurisdictional claims in published maps and institutional affiliations.

Copyright: ( $\odot 2021$ by the authors. Licensee MDPI, Basel, Switzerland. This article is an open access article distributed under the terms and conditions of the Creative Commons Attribution (CC BY) license (http://creativecommons.org/licenses/by/4.0/).

MDPI AG, St. Alban-Anlage 66, 4052 Basel, Switzerland

Peer review is the driving force of journal development, and reviewers are gatekeepers who ensure that Multimodal Technologies and Interaction maintains its standards for the high quality of its published papers. Thanks to the cooperation of our reviewers, in 2020, the median time to first decision was 23 days and the median time to publication was 55.5 days. The editors would like to express their sincere gratitude to the following reviewers for their precious time and dedication, regardless of whether the papers were finally published:

Afzal, Muhammad Raheel

Aguilar, Stephen J.

Ahmad, Muneeb Imtiaz

Albertos, Felix

Alenljung, Beatrice

Alimardani, Maryam

Allison, Fraser

Altmeyer, Maximilian

Anderson, Allie

Ang, Eng Tat

Aylward, Katie

Azadegan, Aida

Babur, Funda Durupinar

Bajenaru, Lidia

Balatsoukas, Panos

Ball, Christopher

Barakova, Emilia

Barkaoui, Kamel

Barreda-Ángeles, Miguel

Barresi, Giacinto

Barreto, Mary

Bera, Aniket

Bertel, Lykke

Billingsley, Glenna

Bliemel, Michael

Boboc, Marius

Bonanad, Santiago

Bozomitu, Radu Gabriel

Bray, Aibhin

Brusaporci, Stefano

Buckingham, Gavin

Burmester, Michael
Burrows, Alison

Byrne, Ceara

Canazza, Sergio

Cappagli, Giulia

Carbone, Marina

Carrera, Carlos Carbonell

Caruso, Giandomenico

Casado-Mansilla, Diego

Casas, Sergio

Castillo, José Manuel Morales Del

Ceccacci, Silvia

Cecchinato, Marta E.

Chandrasegaran, Senthil

Chaudhry, Sohail S.

Cheverst, Keith

Chinthammit, Winyu

Cibrian, Franceli L.

Coghlan, Alexandra

Coiera, Enrico

Colley, Ashley

Cordeiro, Mário

Cortés, Rocío Jiménez

Crisol-Moya, Emilio

Croce, Daniele

Croy, Glen

Cullen, Theresa

Cunliffe, Daniel

Cunningham, Stuart

Da Silva, Hugo Plácido

Davila, Maria I.

De Carolis, Berardina

DeCristofaro, Claire 
Del Mastro, Franca

Delle Monache, Stefano

Desharnais, Jean-Marc

Diago, Pascual D.

Diao, Pei-Huang

Dmitrenko, Dmitrijs

Dondi, Piercarlo

Dondrup, Christian

Edyburn, Dave

Eigen, David

Engström, Henrik

Engwall, Olov

Escudero, David

Eskridge, Thomas C.

Esteve, Francesc

Fakhr Hosseini, Shabnam

Farhadi, Alireza

Feijs, Loe M.G.

Filippi, Stefano

Forster, Yannick

French, Fiona

Fuhrmann, Arnulph

Gaffar, Vanessa

Garay-Vitoria, Nestor

García-Valcárcel Muñoz-Repiso, Ana

Gardecki, Arkadiusz

Garner, Tom

Garrido-Hidalgo, Celia

Gay, Simon

Gesi, Marco

Ghajargar, Maliheh

Giannakas, Filippos

Gil-Iranzo, Rosa

Gillespie, R. Brent

Giudice, Nicholas A.

Göritz, Anja

Gouranton, Valérie

Greiff, Samuel

Grincheva, Natalia

Gürdür Broo, Didem

Hakulinen, Jaakko

Hall, Peter

Ham, Jaap

Hampton, Andrew J.

Hannukainen, Pia

Hansen, Christian

Hartley, Kendall

Hegde, Sudeep

Henshaw, Alexis

Herman, Lukáš

Hilbert, Sven
Hitz, Martin

Hollis, Victoria

Horvat, Christopher

Hou, Shangguo

Hovenga, Evelyn

Hubinsky, Peter

Hussain, Jamil

Hussmann, Heinrich

Iannello, Paola

Imai, Michita

Isernia, Sara

Jackson, Thomas W.

Jiang, Yalong

Johansson, Fredrik

Johnson, Jeff

Jonason, Peter

Jones, Ray

Jonkman, Mirjam

Jung, Sungchul

Kafetsios, Konstantinos

Kaipainen, Kirsikka

Kanthawala, Shaheen

Kasnesis, Panagiotis

Katz, Brian FG

Kawase, Satoshi

Ke, Fengfeng

Kelliher, Aisling

Keshtkar, Fazel

Khalil, Mohammad

Khan, Masood Mehmood

Kiefer, Chris

Kim, Youngjoo

Kimball, Miles A.

King, Scott A.

Klimova, Blanka

Kocaballi, A. Baki

Koch, Katherine

Koleva, Boriana

Konkani, Avinash

Korb, Werner

Kotis, Konstantinos

Kotropoulos, Constantine

Kouroupetroglou, Georgios

Koustoumpardis, Panagiotis

Kraak, Menno-Jan

Kraus, Martin

Krebs, Claudia

Krems, Josef

Kruusamäe, Karl

Kulshreshth, Arun

Lankes, Michael 
Laschke, Matthias

Lavicza, Zsolt

Lech, Michal

Li, Jamy

Li, Xiaomeng

Liao, Yabin

Liarokapis, Fotis

Lieberoth, Andreas

Lindblom, Jessica

Liu, Kai-Chun

Lok, Benjamin

Lopez, Jose J.

López, Manuela

Lorenzo Lledó, Alejandro

Lorenzo, Gonzalo

Machado Alexandre, Isabel

Machulla, Tonja

Maguire, Martin

Mahmoudzadeh, Ahmadreza

Maike, Vanessa R. M. L.

Majchrzak, Tim A.

Malekigorji, Maryam

Mancini, Clara

Manning, Jimmie

Marconi, Stefania

Marcu, Gabriela

Margetis, George

Martelaro, Nik

Matu, Silviu Andrei

Mayer, Sven

McGowan, John

Mędrzycka, Wioletta

Meißner, Martin

Mekler, Elisa

Melcer, Edward F.

Merino, Leonel

Miguel-Revilla, Diego

Mingorance-Estrada, Ángel Custodio

Mitsogiannis, Iraklis

Mlakar, Uroš

Mohino, Inma

Moldovan, Arghir-Nicolae

Montanari, Rebecca

Monteleone, Salvatore

Morais, Diogo

Moreno-Vera, Juan-Ramón

Morreale, Fabio

Mostafid, Hugh

Mota, José Miguel

Moulianitis, Vassilis C.

Mueller, Wolfgang
Munafo, Andrea

Muñoz-Saavedra, Luis

Nalbone, David

Neves, António J. R.

Nichols, Jeffrey

Niewiadomski, Adam

Nijeweme-d'Hollosy, Wendy Oude

Nikos, Antonopoulos

Nofal, Eslam

Noori, Farzan Majeed

Nordström, Thomas

Nowak, Beata

Nykänen, Arne

O'Shaughnessy, Douglas

Ohta, Naohisa

Opdenakker, Raymond J.G.

Orehovački, Tihomir

Orlosky, Jason

Östlund, Britt

Owens, David C.

Oyibo, Kiemute

Papadakis, Stamatios

Papangelis, Konstantinos

Passig, David

Payo, Valentín Cardeñoso

Peñarroja-Cabañero, Vicente

Perry, Patsy

Persson, Johanna

Pesquita, Catia

Peters, Dorian

Petersson, Göran

Petreca, Bruna

Picazo, Patricia

Pilato, Giovanni

Pill, Shane

Piras, Andrea

Pirbhulal, Sandeep

Poce, Antonella

Poppen, Marcus

Potenziani, Marco

Poushneh, Atieh

Predic, Bratislav

Price, Sara

Prior, Suzanne

Prophet, Jane

Rahimi, Ebrahim

Rahimian, Farzad Pour

Rak, Massimiliano

Randell-Moon, Holly

Regan, Elizabeth A.

Reichert, Christoph 
Reinertsen, Anne Beate

Reinhold, Frank

Reysen, Stephen

Richards, Deborah

Rinta, Tiija

Riva, Silvia

Robert, Lionel

Roberts, David L.

Robinson, Charlotte

Rodríguez Baena, Luis

Romero, Francisco P.

Ron-Angevin, Ricardo

Ros, Raquel

Rosquete, Daniel

Ruiz, Manuel Armayones

Ruser, Heinrich

Sadjadi, Firooz

Sánchez Fernández, María Dolores

Sandoval, Juan

Savino, Sergio

Sayem, Abu Sadat Muhammad

Schneider, Oliver

Schroeder, Sascha

Schubert, Gerhard

Schulze, Julian

Schuurman, Dimitri

Seaman, Sean

Seo, Jinsil

Shafer, Daniel M.

Sharf, Inna

Sherman, Paul

Shi, Lei

Shlomo, Berkovsky

Siemon, Dominik

Silva, Bruno

Simons, Joseph JP

Sintoris, Christos

Smith, Melody

Söbke, Heinrich

Spano, Lucio Davide

Spiliotopoulos, Dimitris

Stadler, Sebastian
Stavrakis, Modestos

Stock-Homburg, Ruth

Stojmenova, Emilija

Sturm, Janienke

Suárez-Carballo, Fernando

Subbanna, Nagesh

Suhartono, Derwin

Svetieva, Elena

Tavernise, Assunta

Theron, Roberto

Thieringer, Florian M.

Tkalcic, Marko

Todolí-Ferrandis, David

Tomitsch, Martin

True Accord, Vladimir Iglovikov

Trujillo, Amaury

Turunen, Markku

Urbaniak, Maciej

Van Den Berghe, Rianne

Van Der Bilt, Andries

Van Eck, Richard

Vatavu, Radu-Daniel

Velikanov, Vladimir

Vendrell-Herrero, Ferran

Verenikina, Irina

Vijayan, Karthika

Vosinakis, Spyros

Wang, Ziran

Wei, Xiu-Shen

Wendrich, Robert

Wethington, Elaine

Wilkerson, John D.

Wilmering, Thomas

Withana, Anusha

Wong, Meng Ee

Woo, Hanseung

Wulff, Dirk U.

Wurhofer, Daniela

Yang, Jing

Yurtman, Aras

Zaman, Bieke

Zhao, Chunying 